\title{
The shape of a hole and that of the surface-with-hole cannot be analyzed separately
}

\author{
Marco Bertamini • Mai Salah Helmy
}

Published online: 12 May 2012

(C) Psychonomic Society, Inc. 2012

\begin{abstract}
Figure-ground organization has a central role in visual perception, since it creates the regions to which properties, such as shape descriptions, are then assigned. However, there is disagreement on how much shape analysis is independent of figure-ground. The reversal of figure-ground of a single closed region is the purest form of figure-ground organization, and the two resulting percepts are that of an object and that of a hole. Both object and hole are nonaccidental regions and can share an identical outline. We devised a test of how figure-ground and contour ownership dramatically affect how shape is processed. Observers judged the shape of a contour that could be either the same as or different from an irrelevant surrounding contour. We report that different (incongruent) inside and outside contours produce a stronger interference effect when they form a single object-with-hole, as compared with a hierarchical set of surfaces or a single hole separating different surfaces (a trench). We conclude that (1) which surface owns the contour constrains the interference between shapes and that (2) despite some recent claims, holes do not display objectlike properties.
\end{abstract}

Keywords Contour ownership · Holes $\cdot$ Shape perception Figure-ground organization

Contours carry information about solid shape, as is easily demonstrated by considering line drawings. Contours tend to be perceived as borders of surfaces, and they describe the surface itself and not the ground region. This principle is known as unidirectional contour ownership (Koffka, 1935; Nakayama, Shimojo, \& Silverman, 1989; Rubin, 1921).

M. Bertamini $(\bowtie) \cdot$ M. S. Helmy

Department of Experimental Psychology, University of Liverpool, Liverpool, England, UK

e-mail: M.Bertamini@liv.ac.uk
Knowing what is figure and what is ground is fundamental for vision to work properly, as is demonstrated by cases of agnosia in which the process is disrupted (Humphreys, 1999). Much work whose aim was to understand figure-ground organization has been conducted, starting from the beginning of the last century (Koffka, 1935; Rubin 1921, 1958), and it is not confined to psychology; contour ownership plays a part in understanding receptive fields of neurons that analyze shape (Lamme, Zipser, \& Spekreijse, 2002; Zhou, Friedman, \& von der Heydt, 2000), and figure-ground segmentation is important in computational vision (Tek \& Kimia, 1995).

An important debate in the figure-ground literature relates to the analysis of the shape of a ground region. Peterson and her colleagues (for a review, see Peterson \& Skow-Grant, 2003) have argued that prior to figure assignment, both sides of a contour are evaluated in terms of configural cues (convexity, closure, symmetry) and familiarity (previously seen objects). Foreground and background, therefore, share an early stage in which both regions are processed in terms of shape and, in particular, past experience influences figureground organization. More relevant for this article, Peterson (2003) has also speculated that in the case of apertures, it is possible to obtain figure-figure segmentation where both regions acquire figural status.

To shed new light on the nature of figural assignment, we measured how quickly observers can classify the shape of a simple configuration perceived as either an object (figure) or a hole (ground). Holes are ideal stimuli for studying the figure-ground phenomenon (Bertamini, 2006), and several authors have used them in recent years (Albrecht, List, \& Robertson, 2008; Horowitz \& Kuzmova, 2011; Nelson \& Palmer, 2001; Peterson, 2003; Zhou, Luo, Zhou, Zhuo \& Chen 2010). However, some studies have found similarities between perception of objects and of holes, while others have found differences. We review this literature next. 
Palmer discussed in his classic book what he called the paradox of holes, because they are ground regions that, in some cases, behave as "figures for purposes of describing shape" (Palmer, 1999, p. 287). The evidence for this comes from studies showing that the shape of holes is remembered as well as that of objects (Nelson, Thierman, \& Palmer, 2009; Palmer, Davis, Nelson, \& Rock, 2008). Importantly, memory for holes is good only for intrinsic holes - that is, holes belonging to a single surface - and not for ground regions defined by a collection of contours from multiple surfaces (an accidental closed contour). Perhaps holes are encoded as their material counterparts, but with an additional empty tag. On the basis of Palmer's findings, Feldman and Singh (2005) suggested that holes are "given a quasi-figural status, as far as shape analysis is concerned" (p. 248).

The idea that holes are unique has important consequences, since it implies that shape analysis - for instance, the coding of convexity and concavity - proceeds relative to the hole and not, or not only, relative to the object-with-hole. Feldman and Singh (2005) were specifically focusing on convexity in their article. Empirical evidence, however, does not support this proposal, because convexity effects do depend on whether a region is perceived as figure or as hole. In a series of studies, Bertamini and colleagues (Bertamini, 2001; Bertamini \& Croucher, 2003; Bertamini \& Farrant, 2006; Bertamini \& Mosca, 2004) have found faster processing of convex than of concave vertices. This was true for objects and was still true, but with a reversal of which vertices were perceived as convex, for holes.

When a location inside a hole is cued, results suggest that what is cued is the surface visible through the hole, as would be expected given that this is a ground region (Albrecht et al., 2008). When observers perform a visual search, there is little difference between searching among holes or among objects (Bertamini \& Lawson, 2006), except for complex shapes, where different coding of part structure, influenced by convexity coding, plays a role (Hulleman \& Humphreys, 2005; they used C-shapes that have both convexities and concavities). Finally, a recent study by Horowitz and Kuzmova (2011) has studied tracking of multiple objects or multiple holes. They concluded that holes can be tracked and are, therefore, protoobjects. These are bundles of features that serve as tokens to which attention can be deployed (Wolfe \& Bennett, 1997).

We investigated the role of figure-ground organization for a task that required a simple shape analysis: to decide whether a region was a square or a circle ( $\sim 3^{\circ}$ of visual angle). In this task, shape recognition could be performed on luminance contours and independently of figure-ground assignment. Any effect of figure-ground relations would depend on information that is processed automatically. The critical region was presented inside another region with an outline that was either a square or a circle (Fig. 1). When both regions were the same, we expected facilitation (faster responses), and when they were different, we expected interference (slower responses).
This logic is adapted from the flanker procedure (Eriksen \& Schultz, 1979). In a typical flanker task, observers respond to a target flanked by distractors. In a congruent trial, the target and the flankers are associated with the same response, but in an incongruent trial, the target and the flankers are associated with a competing response. Because flankers lead to activation of the response associated with these stimuli, response times are longer on incongruent than on congruent trials. Figure 2 shows examples where the surrounding shape was either the same as (congruent) or different from (incongruent) the central region.

Unlike other studies, our methodology allows a test of whether shape analysis can proceed independently of figure-ground organization, because the task is about shape but figural relationships are task irrelevant. In addition, there is no role for memory, avoiding the problem that a judgment about the shape of holes could be based on the memory for the object-with-hole.

Because the region perceived as either object or hole was the same, in terms of spatial attention the same region of space had to be attended. However, depth stratification may be a factor. Many studies have reported that attention can be allocated in depth, although this effect requires a high perceptual load and, typically, a number of distractors (Arnott \& Shedden, 2000; Atchley, Kramer, Andersen, \& Theeuwes, 1997). In our displays, depth was ordinal, and the difference was the direction of separation between surfaces: The object was a region nearer the observer, and the hole was a region farther away. We return to the issue of location in depth in Experiment 2.

In Experiment 1a, texture and shading created a sense of surface layout. We then extended the findings to surface layout specified by shading alone (Experiment $1 \mathrm{~b}$ ) and by a minimal amount of shading (Experiment 1c). In Experiment 2, we changed depth relations so that the target surface was coplanar with the background and separated from it by a trench. In Experiment 3, to reduce speed differences between conditions, we presented holes nearer fixation and objects farther, in one of four quadrants. Finally, in Experiment 4, observers performed an additional task: After the shape judgment, they reported whether they had seen an object or a hole. In all cases, a stronger interference from the outside shape on the perception of the inside shape was present when both contours (inside and outside) belonged to the same surface.

\section{General method}

Stimuli The stimuli were presented using a Macintosh computer and a Sony monitor (resolution, 1,024 × 768; $85 \mathrm{~Hz}$ ). Presentation was controlled by a program written in $\mathrm{C}++$ and OpenGL. The vertical heights were $8 \mathrm{~cm}$ for the background (16 cm in Experiment 3), $6 \mathrm{~cm}$ for the stimulus, and 
Fig. 1 The structure of a single trial: After a fixation screen, the stimulus is presented either on the left or on the right side. This example shows the congruent condition in Experiment 1, because both inside and outside regions are squares. What is shown is a hole condition in which the two regions formed an object-with-hole. In the object condition, the two regions would, instead, appear as one in front of the other. The dark outside background extended to the whole screen
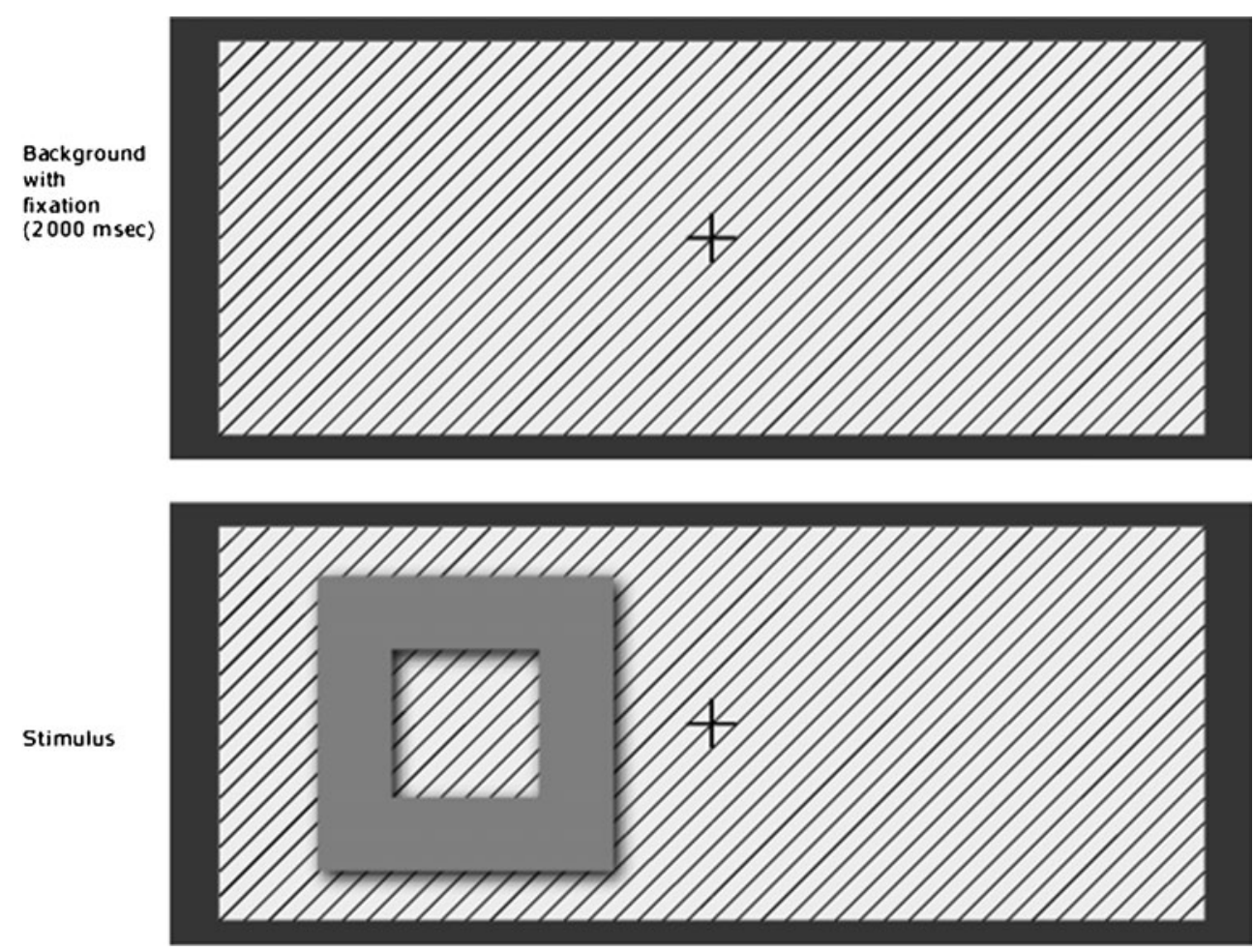

$3 \mathrm{~cm}$ for the central region. Horizontally, the total length of the background was $16 \mathrm{~cm}$ (Fig. 1). The shading was generated by a Gaussian-blurred black region displaced toward the lower right corner by $14 \mathrm{~mm}$, except in Experiment 1c, where the displacement was $7 \mathrm{~mm}$. This corresponds to lighting from the top left, which is the preferred direction for human observers (Mamassian \& Goutcher, 2001). The only source of illumination in the room was a light placed on the left side and higher than the monitor, so that lighting and direction of shading were consistent.

Design The factors were the shape of the central region (square or circle), congruency (the shapes of the inside and outside regions were the same or different), objectness (objects or holes), and location of the stimulus (left and right). The dark shadows were more on the right when the central region was an object, as compared with when it was a hole. Presenting the stimulus to the left and to the right allows a test of the role of this asymmetry: If shadow location matters, objectness should interact with location. In Experiment 4, the stimuli were presented centrally, but they remained visible only for $200 \mathrm{~ms}$.

Participants In each version of the experiment, there were 10 participants from the University of Liverpool community, except in Experiment 2 (20) and in Experiment 4 (15). They had normal or corrected-to-normal vision. Students received course credit for their time.
Procedure Observers sat in a quiet room at approximately $57 \mathrm{~cm}$ from the monitor. During a trial, a fixation cross was presented for $2 \mathrm{~s}$; next, the stimulus was presented $4 \mathrm{~cm}$ to the left or to the right of fixation. In Experiment 3, this same offset was both horizontal and vertical, placing the objects in one of four quadrants, while the offset was reduced to $1 \mathrm{~cm}$ for the holes. In Experiments 1-3, the stimulus remained visible until the participant had responded. In Experiment 4, presentation time was $200 \mathrm{~ms}$. Participants pressed one of two keys to indicate that the shape was a square or a circle. In Experiment 4 , this speeded response was followed by a second task, prompted by a question on the screen, in which observers reported whether they had seen an object or a hole. Each experiment started with 15 practice trials, after which participants confirmed that the instructions were clear to them, followed by 192 experimental trials. Practice trials were identical in design to experimental trials but were excluded from the analysis. After each block of 64 trials, a message appeared, and the observer was allowed time to rest. The start of the subsequent blocks was self-paced.

\section{Results}

Experiment 1a As is shown in Fig. 1, the surface behind the central object was gray with a pattern of diagonal lines. Orientation of the lines within the central region was reversed in the object condition (Fig. 2), because similarity in texture over the background contributes to the perception of holes 


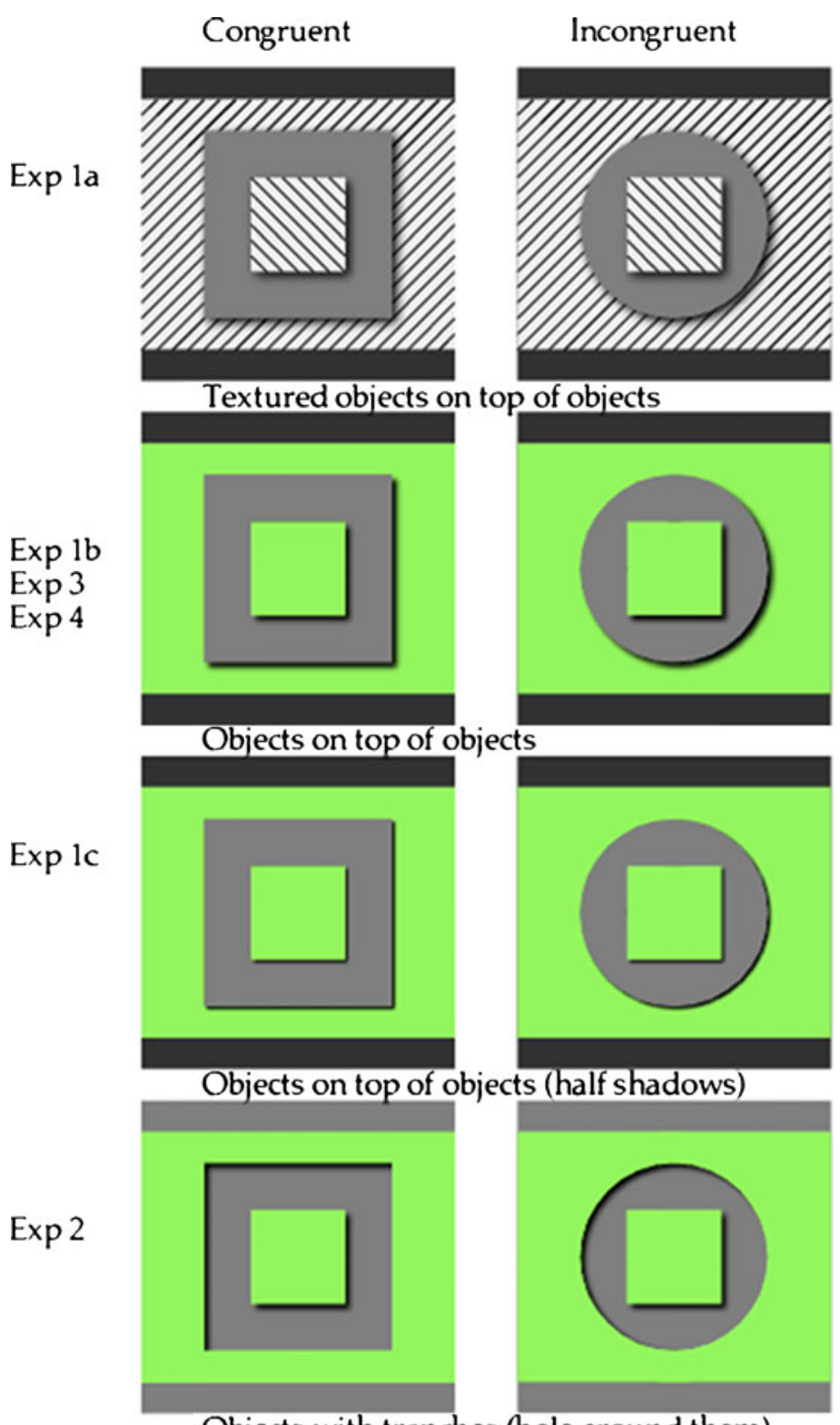

Objects with trenches (hole around them)

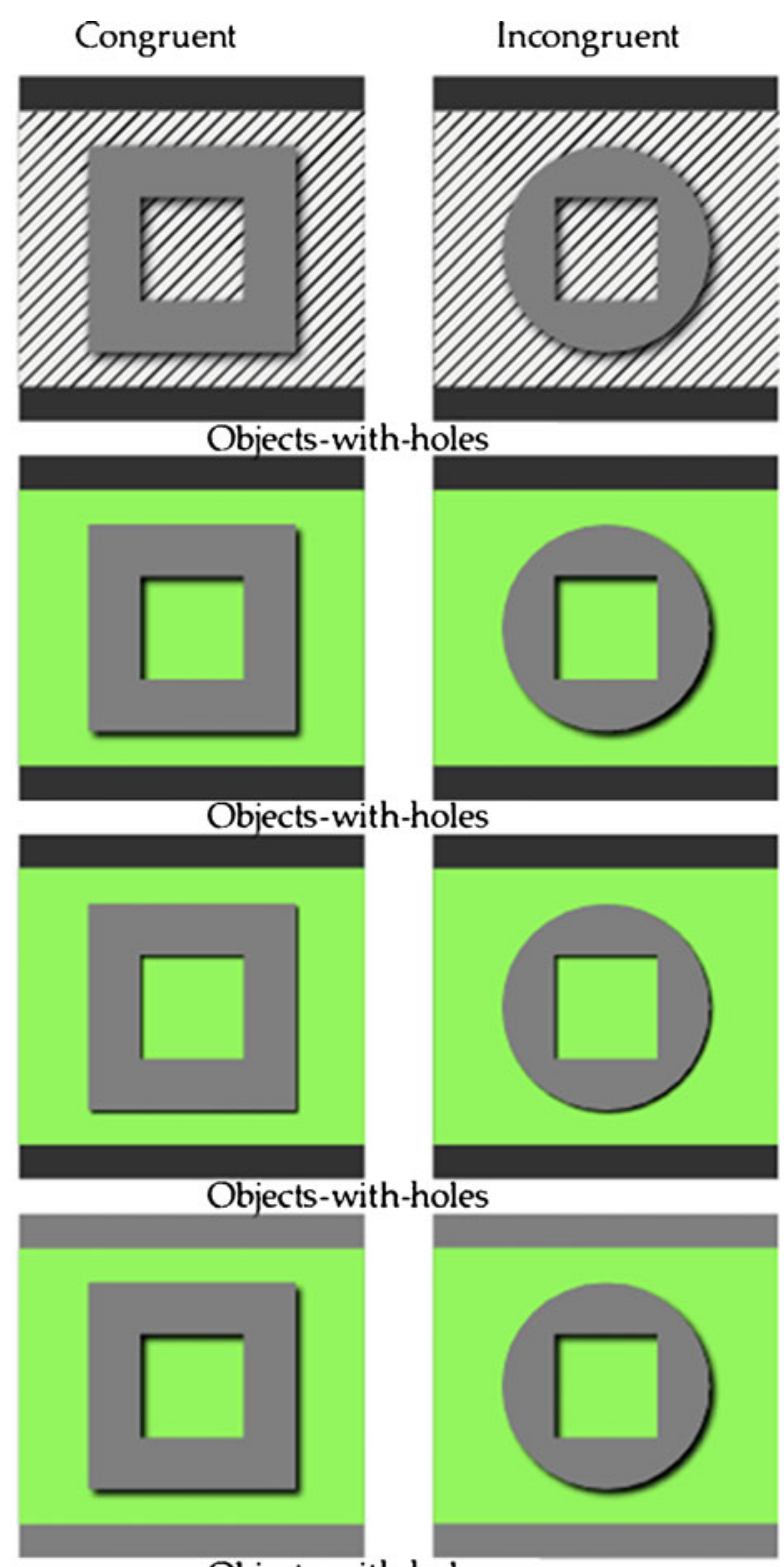

Objects-with-holes
Fig. 2 Examples of stimuli used in all experiments. For each condition, each pair of images shows a congruent stimulus (on the left) and an incongruent stimulus (on the right). For reasons of space, only the examples in which the central region is a square are shown. In another

(Nelson \& Palmer, 2001). The orientation difference was $90^{\circ}$, and overall brightness and contrast in this region did not vary with orientation.

Error trials and outliers (response times more than $3 S D \mathrm{~s}$ from the mean) were excluded from the analysis. The excluded data were $2.8 \%$ of the total. Figure 3 shows response times and error rates. Although low, for comparison, the error rates are reported at the bottom of the graph for each condition.

We performed a repeated measures ANOVA with objectness (object and hole), congruency (congruent and incongruent), set, the central region was a circle. A small portion of the outside background is shown above and below the stimulus; this extended to the whole screen. This color was changed in Experiment 2 to be compatible with a trench interpretation

and location (left and right) as factors. ${ }^{1}$ There was an effect of objectness [faster responses to objects, $F(1,9)=28.21$, $p<.001$, partial $\eta^{2}=.76$ ], an effect of congruency [faster responses in the congruent condition, $F(1,9)=30.77$, $p<.001$, partial $\left.\eta^{2}=.77\right]$, and an interaction between objectness and congruency, $F(1,9)=18.72, p=.002$, partial $\eta^{2}=.68$. This interaction is due to the presence of a

\footnotetext{
${ }^{1}$ We report the analysis of mean response time, but the same pattern of results was present when the medians were used instead of the means.
} 


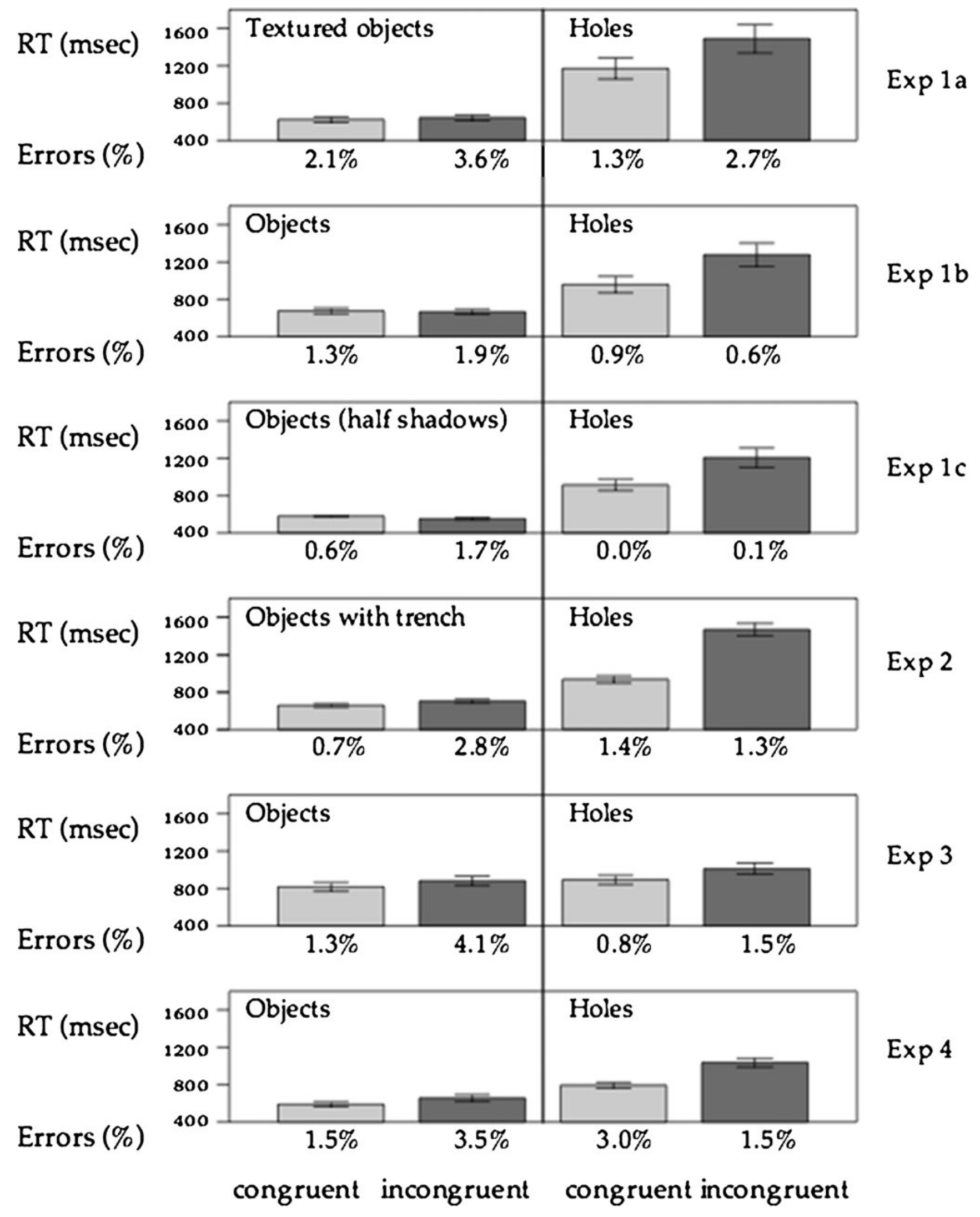

Fig. 3 For each experiment, the graphs show mean response time (RTs) for congruent (on the left, light gray) and incongruent (on the right, dark gray) stimuli. Error bars are $\pm 1 S E M$. Error rates are reported underneath each condition 
larger difference between congruent and incongruent conditions for holes. This can be described as an interference effect and is consistent with the hypothesis that holes cannot be processed independently of the surface that they belong to.

Experiment $1 b$ This version relied on shading alone, and not on changes in texture, to specify foreground and background regions on top of a uniform green surface (Fig. 2). Error trials and outliers (response times more than $3 S D$ s from the mean) were excluded from the analysis (2.2\%). A repeated measures ANOVA with objectness, congruency, and location as factors confirmed a significant effect of objectness [faster responses to objects, $F(1,9)=27.43, p=.001$, partial $\eta^{2}=.75$ ], an effect of congruency [faster responses in the congruent condition, $F(1,9)=22.42, p=.001$, partial $\left.\eta^{2}=.71\right]$, and an interaction between objectness and congruency, $F(1,9)=$ $30.79, p<.001$, partial $\eta^{2}=.77$. Therefore, Experiments $1 \mathrm{a}$ and $1 \mathrm{~b}$ confirmed the same pattern of interference, with a difference between hole and object conditions.

Experiment $1 c$ In Experiment $1 \mathrm{~b}$, we found that shading information was enough to specify stratification, without a texture. In Experiment 1c, we reduced the length of the shadow by half (Fig. 2) to test whether this weaker version would produce the same pattern. Error trials and outliers (response times more than $3 S D$ s from the mean) were excluded from the analysis $(1.7 \%)$. We performed a repeated measures ANOVA with objectness, congruency, and location as factors. There was a significant effect of objectness [faster responses to objects, $F(1,9)=32.13, p<.001$, partial $\eta^{2}=.78$ ], an effect of congruency [faster responses in the congruent condition, $F(1,9)=38.44, p<.001$, partial $\eta^{2}=.81$, and an interaction between objectness and congruency, $F(1,9)=30.47, p<.001$, partial $\eta^{2}=.77$. This is the same pattern as in Experiments 1a and $1 \mathrm{~b}$.

Combined analysis We performed a mixed ANOVA with objectness, congruency, and location as within-subjects factors and version $(\mathrm{a}, \mathrm{b}, \mathrm{c})$ as a between-subjects factor. There was an effect of objectness [faster responses to objects, $F(1,27)=82.23, p<.001$, partial $\left.\eta^{2}=.75\right]$, an effect of congruency [faster responses in the congruent condition, $F(1,27)=86.01, p<.001$, partial $\left.\eta^{2}=.76\right]$ an effect of location, $F(1,27)=6.07, p<.05$, partial $\eta^{2}=0.18$, and an interaction between objectness and congruency, $F(1,27)=$ $76.31, p<.001$, partial $\eta^{2}=.74$. Because the interference was stronger in the hole condition, we conclude that holes cannot be processed independently of the surface that they belong to. A hole region cannot be treated as figural, even when contour ownership is task irrelevant.
Experiment 2 Experiment 2 tested the hypothesis that the interference emerges when contours are perceived as located on the same depth plane, since this might explain the weaker interference for the objects in Experiment 1. Therefore, we created objects that appeared to be on the same depth plane as the outside region by defining a trench around them. This is a type of hole, but importantly the inside contour and the outside contour are not owned by the same surface. If one compares the location in depth of the central surface in the two conditions, this green region is on the same depth plane for both trench and hole conditions (Fig. 2). We expected the hole condition to produce greater interference because, in that case, the relevant and irrelevant contours belonged to the same surface. In addition, Experiment 2 also tested whether the presence of a hole in itself is the reason for the interference: Both conditions included holes, but we predicted that interference would be weaker when the hole formed a trench.

Error trials and outliers (response times more than $3 S D \mathrm{~s}$ from the mean) were excluded from the analysis (1.7\%). We performed a repeated measures ANOVA with objectness (trenches and holes), congruency, and location as withinsubjects factors. There was a significant effect of objectness [faster responses to trenches, $F(1,19)=231.03, p<.001$, partial $\eta^{2}=.93$ ], an effect of congruency [faster responses in the congruent condition, $F(1,19)=246.05, p<.001$, partial $\left.\eta^{2}=.92\right]$, and an interaction between the two, $F(1,19)=$ 134.75, $p<.001$, partial $\eta^{2}=.88$. This analysis, therefore, confirmed an interaction between objectness and congruency as in the previous experiments. We interpret this as the result of a difference in the relationship between contours: In the hole condition, but not in the trench condition, the inside and outside contours belong to the same surface.

Experiment 3 One interesting aspect of the results is a significantly slower response to holes than to objects. This is consistent with the hypothesis that, in the case of holes, the outside shape must be processed and, therefore, the system has more information to analyze. Nevertheless, we decided to try and reduce the overall difference in response time. Experiment 3 was an attempt to do so while not changing the shapes, which were matched on several important dimensions (shape, size, luminance, and contrast). We used the same stimuli and design as that in Experiment $1 \mathrm{~b}$ but presented the hole stimuli with a smaller offset from fixation, as compared with the object stimuli. The $1-\mathrm{cm}$ offset for the holes meant that the central region overlapped the location of the fixation cross. The offset for the object, on the other hand, was the same as in Experiment 1 but was now both horizontal and vertical. The green background 
was changed to a square, and the object stimulus could therefore appear in one of the four quadrants of this square.

Error trials and outliers (response times more than $3 S D \mathrm{~s}$ from the mean) were excluded from the analysis (3.3\% of the total). We performed an ANOVA with objectness, congruency, and location as factors. The analysis confirmed a significant effect of objectness [despite the changes in eccentricity, responses to objects were faster, $F(1,9)=20.30$, $p=.001$, partial $\eta^{2}=.69$ ], an effect of congruency [faster responses in the congruent condition, $F(1,9)=33.75, p<.001$, partial $\eta^{2}=.79$ ], and an interaction between objectness and congruency, $F(1,9)=10.41, p=.010$, partial $\eta^{2}=.54$. Responses to objects were still faster than responses to holes, but when compared with Experiment 1b, they were slower than those in the object condition, $t(18)=3.17, p=.005$, and not different from those in the hole condition, $t(18)=1.94$, n.s. Experiment 3 confirmed the interaction between objectness and congruency even though the means were in a different range, as compared with Experiment $1 \mathrm{~b}$.

Experiment 4 On each trial, observers were asked for a response about shape, just as in the other experiments, but afterward they reported whether the region that they had just seen was an object or a hole. This second response was not timed. The stimuli were the same as those in Experiment 1b, but they were presented near fixation. Unlike in the previous experiments, they disappeared after $200 \mathrm{~ms}$, thus precluding multiple fixations on the stimulus. We did this to test whether a single glance at the stimulus was sufficient to produce the effect. One possibility is that the long response times for holes might have been caused by a different pattern of exploratory eye movements, which, in turn, might be related to the interference, perhaps because observers' fixation is directed at the outside of the object-with-hole.

Error trials and outliers (response times more than $3 S D \mathrm{~s}$ from the mean) were excluded from the analysis (3.9\%). We performed an ANOVA with objectness and congruency as factors. There was a significant effect of objectness [faster responses to objects, $F(1,14)=86.64, p<.001$, partial $\eta^{2}=.86$ ], an effect of congruency [faster responses in the congruent condition, $F(1,14)=108.54, p<.001$, partial $\left.\eta^{2}=.88\right]$, and an interaction between objectness and congruency, $F(1,14)=39.01, p<.001$, partial $\eta^{2}=.74$.

On the second task, participants performed well, with only $4.9 \%$ errors. This is reassuring since it confirms that holes were perceived as holes and objects as objects. The low percentage makes it difficult to analyze this subset on its own, but we repeated the ANOVA with these trials excluded and confirmed the interaction, $F(1,14)=114.39, p<.001$, with a larger effect size (partial $\eta^{2}=.89$ ). This supports the idea that the percept of figure-ground is related to the interaction.

\section{Conclusions}

We have used a simple task involving the discrimination between a circle and a square. Congruent and incongruent conditions were created by using outside regions that were either circular or square. As was expected, responses were slower in the incongruent than in the congruent condition (interference), but in all experiments, this effect was stronger when inside and outside contours belonged to the same surface. This suggests that surfaces are computed quickly and that ownership of the contour affects shape processing. The task of discriminating between a circle and a square is easy and can be performed on the basis of basic features, such as size and line terminators. Therefore, in our experiments, it is possible to respond to the onset of the target without focused attention. The implication is that a hole and an object do not share the same preattentive representation (proto-objects) but are, instead, different as far as shape analysis is concerned. This evidence, therefore, is incompatible with recent claims that the shape of holes is analyzed by the visual system as if they were figures (Feldman \& Singh, 2005; Palmer et al., 2008) or that holes behave as proto-objects (Horowitz \& Kuzmova, 2011).

Changes of texture (Experiment 1a vs. 1b) or of the extent of the shadow (Experiment 1b vs. 1c) had little impact on the results. Interference was stronger for holes even when they were contrasted to trenches: a stimulus in which, similarly to the hole condition, all relevant contours are perceived as coplanar (Experiment 2). The interaction was present even when holes were presented near fixation and responses were, therefore, faster (Experiment 3) and when stimuli were presented for only $200 \mathrm{~ms}$ (Experiment 4).

These results are consistent with the idea that object segmentation - and in particular, contour ownership - is the critical factor, not the properties of the surfaces, not a difference in eye movements, and not the extent of the depth separation between foreground and background.

Holes specify locations in space, and attention may be directed to these locations in visual search (Bertamini \& Lawson, 2006) or in multiple object tracking (Horowitz \& Kuzmova, 2011). However, when an analysis of the shape of the region is required, there is no evidence that holes possess figural properties. Holes, therefore, are not an exception to the generality of the principle of unidirectional contour ownership.

In all experiments, response times to holes were slower, as compared with the object (or trench) condition. There are two related reasons for this. The first is that additional shape information has to be processed in the hole condition. This is the information that causes the interference. Second, holes require longer response times because they have a degree of 
ambiguity, caused by the fact that closure is a figural factor, but in the case of a hole, the closed region is not the figure. These two aspects are related because the ambiguity is resolved by an analysis of the object-with-hole or, in other words, when the hole is correctly identified as a property of another surface of which the hole is a local feature.

In the case of interference effects-and specifically, the flanker task-it is known that the interference is strongest for faster responses (Gratton, Coles, \& Donchin, 1992; Stins, Polderman, Boomsma, \& de Geus, 2007). Therefore, it is unlikely that the difference in response time is a mediating factor in generating the effect we report, given that the interference was present for the slower and not the faster condition. Faster responses to objects may also be related to an attentional advantage for the foreground region, a finding that has been reported in many studies (Mazza, Turatto, \& Umiltà, 2005; Nelson \& Palmer, 2007; Wong \& Weisstein, 1982). However, the object-with-hole is a foreground surface, and a foreground advantage in itself cannot explain the interference effect.

Our results are consistent with those of other studies that have compared holes and objects. For instance, Bertamini and Farrant (2006) found evidence that the perceived part structure of a region depends on whether the region is specified as a hole or an object within a random dot stereogram. On a different topic, but also using stereograms, Gillam and Cook (2001) found that, because a trapezoidal hole does not own its contour, its shape has no effect on the perceived slant of the surface seen within that region.

At a philosophical level, Casati and Varzi (1994) have written about the ontology of holes. They used the term "superficialities" to describe holes, and this term nicely captures the strong and unbreakable link between a hole and the surface that it belongs to. If this link is broken, a hole is no longer perceived as a hole. Our results demonstrate this link between the shape of a hole and the shape of the surface-with-hole. Even when the task requires a categorization of the hole region itself, the shape of the hole and that of the surface-with-hole cannot be analyzed separately.

\section{References}

Albrecht, A. R., List, A., \& Robertson, L. C. (2008). Attentional selection and the representation of holes and objects. Journal of Vision, 8(13, Art. 8), 1-10.

Arnott, S. R., \& Shedden, J. M. (2000). Attention switching in depth using random-dot autostereograms: Attention gradient asymmetries. Perception \& Psychophysics, 62, 1459-1473.

Atchley, P., Kramer, A. F., Andersen, G. J., \& Theeuwes, J. (1997). Spatial cuing in a stereoscopic display: Evidence for a "depth-aware" attentional focus. Psychonomic Bulletin \& Review, 4, 524-529.

Bertamini, M. (2001). The importance of being convex: An advantage for convexity when judging position. Perception, 30, 1295-1310.
Bertamini, M. (2006). Who owns the contour of a visual hole. Perception, 35, 883-894.

Bertamini, M. \& Croucher, C. J. (2003). The shape of holes. Cognition, $87,33-54$

Bertamini, M., \& Farrant, T. (2006). The perceived structural shape of thin (wire-like) objects is different from that of silhouettes. Perception, $35,1679-1692$.

Bertamini, M., \& Lawson, R. (2006). Visual search for a circular region perceived as a figure versus as a hole: Evidence of the importance of part structure. Perception \& Psychophysics, 68, 776-791.

Bertamini, M., \& Mosca, F. (2004). Early computation of contour curvature and part structure: Evidence from holes. Perception, $33,35-48$.

Casati, R., \& Varzi, A. C. (1994). Holes and other superficialities. Cambridge, MA: MIT Press.

Eriksen, C. W., \& Schultz, D. W. (1979). Information processing in visual search: A continuous flow conception and experimental results. Perception \& Psychophysics, 25, 249-263.

Feldman, J., \& Singh, M. (2005). Information along contours and object boundaries. Psychological Review, 112, 243-252.

Gillam, B., \& Cook, M. L. (2001). Perspective based on stereopsis and occlusion. Psychological Science, 12, 424-429.

Gratton, G., Coles, M. G. H., \& Donchin, E. (1992). Optimizing the use of information: Strategic control of activation of responses. Journal of Experimental Psychology. General, 121, 480-506.

Horowitz, T. S., \& Kuzmova, Y. (2011). Can we track holes? Vision Research, 51, 1013-1021.

Hulleman, J., \& Humphreys, G. W. (2005). The difference between searching amongst objects and searching amongst holes. Perception \& Psychophysics, 67, 469-482.

Humphreys, G. W. (1999). Integrative agnosia. In G. W. Humphreys (Ed.), Case studies in the neuropsychology of vision (pp. 41-58). London: Psychology Press.

Koffka, K. (1935). Principles of Gestalt psychology. New York: Harcourt Brace.

Lamme, V. A. F., Zipser, K., \& Spekreijse, H. (2002). Masking interrupts figure-ground signals in V1. Journal of Cognitive Neuroscience, 14, 1044-1053.

Mamassian, P., \& Goutcher, R. (2001). Prior knowledge on the illumination position. Cognition, 81, B1-B9.

Mazza, V., Turatto, M., \& Umiltà, C. (2005). Foreground-background segmentation and attention: A change blindness study. Psychological Research, 69, 201-210.

Nakayama, K., Shimojo, S., \& Silverman, G. H. (1989). Stereoscopic depth: Its relation to image segmentation, grouping, and the recognition of occluded objects. Perception, 18, 55-68.

Nelson, R. A., \& Palmer, S. E. (2001). Of holes and wholes: The perception of surrounded regions. Perception, 30, 1213-1226.

Nelson, R. A., \& Palmer, S. E. (2007). Familiar shapes attract attention in figure-ground displays. Perception \& Psychophysics, 69, 382-392.

Nelson, R. A., Thierman, J., \& Palmer, S. E. (2009). Shape memory for intrinsic versus accidental holes. Attention, Perception, \& Psychophysics, 71, 200-206.

Palmer, S. E. (1999). Vision science: From photons to phenomenology. Cambridge, MA: MIT Press.

Palmer, S. E., Davis, J., Nelson, R., \& Rock, I. (2008). Figure-ground effects on shape memory for objects versus holes. Perception, 37 , $1569-1586$.

Peterson, M. A. (2003). On figures, grounds, and varieties of amodal surface completion. In R. Kimchi, M. Behrmann, \& C. Olson (Eds.), Perceptual organization in vision: Behavioral and neural perspectives (pp. 87-116). Mahwah, NJ: Erlbaum.

Peterson, M. A., \& Skow-Grant, E. (2003). Memory and learning in figure-ground perception. In B. Ross \& D. Irwin (Eds.), 
Cognitive vision: Psychology of learning and motivation (pp. 134). San Diego, CA: Academic Press.

Rubin, E. (1921). Visuell wahrgenommene figuren. Copenhagen: Glydenalske boghandel.

Stins, J. F., Polderman, J. C. T., Boomsma, D. I., \& de Geus, E. J. C. (2007). Conditional accuracy in response interference tasks: Evidence from the Eriksen flanker task and the spatial conflict task. Advances in Cognitive Psychology, 3, 389-396.

Tek, H., \& Kimia, B. (1995, June). Image segmentation by reactiondiffusion bubbles. In Proceedings of the 5th International Conference of Computer Vision (pp. 156-162). Cambridge, MA.
Wolfe, J. M., \& Bennett, S. C. (1997). Preattentive object files: Shapeless bundles of basic features. Vision Research, 37, 25-43.

Wong, E., \& Weisstein, N. (1982). A new perceptual context-superiority effect: Line segments are more visible against a figure than against a ground. Science, 218, 587-589.

Zhou, H., Friedman, H. S., \& von der Heydt, R. (2000). Coding of border ownership in monkey visual cortex. Journal of Neuroscience, 20, 6594-6611.

Zhou, K., Luo, H., Zhou, T., Zhuo, Y., \& Chen, L. (2010). Topological change disturbs object continuity in attentive tracking. Proceedings of the National Academy of Sciences, 107, 21920-21924. 